Dhaka Univ. J. Sci. 60(1): 21-24, 2012 (January)

\title{
Continuous Dependence of Fixed Points on Parameters and Initial Conditions
} Md. Rezaul Islam ${ }^{1}$, Razina Ferdousi ${ }^{2}$, Md. Showkat Ali ${ }^{2}$ and M. R. Khan ${ }^{2}$

${ }^{1}$ Department of Science and Humanities, Military Institute of Science and Technology, Mirpur, Dhaka-1000, Bangladesh

${ }^{2}$ Department of Mathematics, University of Dhaka, Dhaka 1000, Bangladesh

Received on 12. 07. 2010. Accepted for Publication on 20. 08. 2011

\begin{abstract}
The main purpose of the paper is to establish conditions for a continuous dependence of fixed points and an application to non-linear functional differential equation of neutral type have been made
\end{abstract}

\section{Introduction}

Functionalanalysis is a promising part of analysis. What is more functional analysis now provides a common language for all areas of mathematics involving the concept of continuity. No serious investigation in the theory of functions, differential equations, or mathematical physics, in numerical methods, mathematical economics or control theory, or innumerous other fields, take place or could take place without extensive use of the language and results of functional analysis. It is precisely this fact that explains on one hand, the rapid development of functional analysis as a mathematical discipline and on the other hand, the everincreasing role played by its techniques in application.

We consider two types of convergence of mappings-point wise convergence and uniform convergence. The theorem regarding the continuity of fixed points of contraction mappings was proved by [3]. Subsequently [9] obtained results concerning sequence of contraction mappings and also gave an application suggested by [7], [8], [9] established conditions implying the strong convergence of the fixed points of a sequence of set-valued contractions. A similar result concerning the weak convergence of the fixed points of the set-valued non-expansive mappings in a Banach space was obtained by [7] who used it to obtain a stability result for generalized differential equations. In our writings we have tried to explain continuous dependence of fixed point sets. The main purpose of the present paper is to establish when the convergence of a sequence of $\phi$ contractive mappings in a uniform space implies a convergence of the sequence of their fixed points. The notion of $\phi$-contractive mapping in a uniform space has been introduced in [2]. In view of the applications given in [2] the problem of a continuous dependence of fixed points can be formulated as a continuous dependence of the solutions of a nonlinear functional differential equation.

To do that we have proved one important theorem regarding fixed points with the help of some lemmas which are also studied.

\section{Main Theorem 1.}

Let $A$ be closed bounded subset of a Hilbert space $H$, d be the norm of $H$ and $D$ the Hausdorff metric on the closed subsets of $A$ generated by $d$. If the family of set-valued maps $F_{k}, k=0,1, \ldots$ satisfy

(1) $F_{k}(x)$ is a non-empty closed convex subsets of $A$ for each $x \in A$.

(2) Each $F_{k}$ is a set-valued contraction, i.e., there is a $k \in[0,1]$ such that

$D\left(F_{k}(x), F_{k}(y)\right) \leq k d(x, y)$ for $x, y \in A$ and $\mathrm{k}=0,1, \ldots$,

(3) $\lim _{k \rightarrow \infty} D\left(F_{k}(x), F_{0}(x)\right)=0$ uniformly for all $x \in A$.

Then the fixed point sets of the sequence $\left\{F_{k}\right\}, k=1,2, \ldots$, converge to the fixed point set of $F_{0}$ in the Housdorff metric $D$.

Before giving the proof of the theorem some lemmas on the closest point projection map associated with $F_{k}$ are required. For $k=0,1, \ldots$, define the maps $F_{k}$ by $f_{k}(x)=$ \{ unique closest point $F_{k}(x)$ to $x$ \} for $x \in A$.

The iterates of each $f_{k}$ are denoted by $f_{k}{ }^{n}, n=2,3, \ldots$, The distance between any $x \in A$ and closed subset $C$ of $A$ will be $d(x, c)=\inf _{c \in C} d(x, c)$.

The following result was given in [5] for a finite dimensional space, but the statement and proof are valid for any Hilbert space.

Lemma 1. If $E$ and $F$ are closed convex subsets of $H$ and $e$ and $f$ are the closest point in $E$ and $F$ to a point $v \in H$, then

$d(e, f) \leq \frac{1}{2}\left(h^{2}+4 h l\right)$, where $h=D(E, F)$ and

$l=d(v, E)$.

For $s \geq 0$ we define the continuous monotone increasing function $g(s)=\frac{1}{2}\left(s^{2}+4 s r\right)$, where $r$ is the diameter of $A$. 
Lemma 2. The maps $f_{k}, k=0,1 \ldots$, are equicontinuous on $A$.

Proof. The family of mappings $f_{k}, k=0,1 \ldots$, will be equicontinuous on $A$ if for every $\varepsilon>0 \quad \exists \delta>0$ such that

$$
d(x, y)<\delta \Rightarrow d\left(f_{k}(x), f_{k}(y)\right)<\varepsilon
$$

Now for any $k=0,1, \ldots$, and $x, y \in A$ let $q$ denote the closest point in $F_{k}(x)$ to $y$.

Then,

$d\left(f_{k}(x), f_{k}(y)\right) \leq d\left(f_{k}(x), q\right)+d\left(q, f_{k}(y)\right)$

The term $d\left(f_{k}(x), q\right)$ is bounded by $d(x, y)$, since projection onto a closed convex set is non-expansive. Since $F_{k}(x), F_{k}(y)$ are non-empty closed convex subsets of $A$ for $x, y \in A$, Lemma 1 implies that $d\left(q, f_{k}(y)\right)$ is bounded by

$$
g\left(D\left(F_{k}(x), F_{k}(y)\right)\right.
$$

Since $F_{k}$ is a set-valued contraction and $g$ is monotone, we have,

$$
g\left(D\left(F_{k}(x), F_{k}(y)\right) \leq g(k d(x, y)) .\right.
$$

The inequality (1) can then be written as

$$
d\left(f_{k}(x), f_{k}(y)\right) \leq d(x, y)+g(k d(x, y))
$$

The map $g$ is continuous and by definition of $g, g(s) \rightarrow 0$ as $s \rightarrow 0$.

Therefore we have,

$$
d\left(f_{k}(x), f_{k}(y)\right) \leq d(x, y)
$$

But $d(x, y)<\delta \Rightarrow d\left(f_{k}(x), f_{k}(y)\right)<\mathcal{E} \quad$ whenever $\delta=\varepsilon$. This completes the proof of the Lemma.

Lemma 3. The sequence of maps $\left\{f_{k}^{n}\right\}, k=0.1, \ldots$, converges uniformly on $A$ to $f_{0}^{n}$ for each $n$.

Proof. For $n=1$ the uniform convergence follows from,

$$
d\left(f_{k}(x), f_{0}(x)\right) \leq g\left(D\left(F_{k}(x), F_{0}(x)\right)\right)
$$

and the uniform convergence of the maps $F_{k}$ to $F_{0}$.

Now we make the induction assumption that $f_{k}^{n-1}, k=1$, $2, \ldots$, converges uniformly on $A$ to $f_{0}^{n-1}$.

From the definition of equi-continuity of $f_{k}$, we have, for $\varepsilon>0$, there exists a $\delta>0$ such that for $u, v \in A$ and $d(u, v)<\delta_{\text {implies }}$

$d\left(f_{k}(u), f_{k}(v)\right)<\varepsilon / 2$ for all $k$

The uniform convergence of the sequence $\left\{f_{k}\right\}$ and $\left\{f_{k}{ }^{n-1}\right\}_{\text {to }} f_{0}$ and $f_{0}{ }^{n-1}$ permits the choice of an integer $N$ such that $k \geq N$ implies

$$
\begin{array}{r}
d\left(f_{k}{ }^{n-1}(x), g_{0}{ }^{n-1}(x)\right)<\delta \\
d\left(f_{k}(x), f_{0}(x)\right)<\mathcal{E} / 2 \quad \forall \quad x \in A .
\end{array}
$$

and

Considering the inequality

$d\left(f_{k}^{n}(x), f_{0}(x)\right)=d\left(f_{k}\left(f_{k}^{n-1}(x), f_{0}^{n-1}(x)\right)\right.$

$\leq d\left(f_{k}\left(f_{k}^{n-1}(x), f_{k}\left(f_{0}^{n-1}(x)\right)+d\left(f_{k}\left(f_{0}^{n-1}(x), f_{0}\left(f_{0}^{n-1}(x)\right)\right.\right.\right.\right.$

$<\varepsilon / 2+\varepsilon / 2=\varepsilon \quad \forall k \geq N$

i. e., $d\left(f_{k}^{n}(x), f_{0}^{n}(x)<\mathcal{E} \quad \forall k \geq N\right.$.

Therefore, the sequence of maps $\left\{f_{k}^{n}\right\}, k=0.1, .$. , converges uniformly on $A$ to $f_{0}^{n}$ for each $n$.

\section{Proof of the Main Theorem 1}

The sequence of iterates $\left\{f_{k}^{n}(x)\right\}_{\text {converges to a fixed }}$ point of $F_{k}$ for $k=0,1, \ldots$, and for all $x \in A$. If $p_{k}$ denotes the fixed point set of $f_{k}$, then from the theorem it follows that

$d\left(f_{k}^{n}(x), p_{k}\right) \leq \sum_{i=1}^{\infty}(r+i) k^{i}$

where $k$ is the Lipschitz constant and $r=d\left(f_{k}(x), p_{k}\right)$.

Each $p_{k}$ is a closed subset and can be written as

$p_{k}=\left\{y \in A: \lim _{n \rightarrow \infty} f_{k}^{n}(x)=y, x \in A\right\}$

Given $\mathcal{E}>0$, choose any $x \in A$ and let

$p_{k}(x)=\lim _{n \rightarrow \infty} f_{k}^{n}(x), k=0.1, \ldots$

Consider the inequality

$d\left(p_{k}(x), p_{0}(x)\right) \leq d\left(p_{k}(x), f_{k}^{n}(x)\right)+$

$d\left(f_{k}^{n}(x), f_{0}^{n}(x)\right)+d\left(f_{0}^{n}(x), p_{0}(x)\right)$

By the estimate (2) we can choose an integer $N$ such that for all $x \in A, d\left(f_{k}^{n}(x), p_{k}(x)\right)<\varepsilon / 3$ for $k=0,1, \ldots$, 
The uniform convergence of $\left\{f_{k}^{N}\right\}$ to $f_{0}^{N}$ permits the choice of an integer $M$ such that $k \geq M$ implies

$$
d\left(f_{k}^{N}(x), f_{0}^{N}(x)\right)<\mathcal{E} / 3 \text { for all } x \in A .
$$

Therefore by inequality (3) becomes

$$
d\left(p_{k}(x), p_{0}(x)\right)<\mathcal{E} \text { for all } x \in A .
$$

Since the points $p_{k}(x)$ range over $p_{k}$ as $x$ ranges over $A$, we have shown that

$$
D\left(p_{k}, p_{0}\right)<\mathcal{E} \text { for } k \geq M .
$$

This proves the convergence of $p_{k}$ to $p_{0}$ in the $D$-metric. Hence the proof is complete.

Theorem 2. [1] Let $(X, A)$ be a locally compact Hausdorff quasicomplete $j$-bounded uniform space. Let $T_{k}: X \rightarrow X$ be a $\phi$-contracted mapping with fixed point

$$
\begin{gathered}
y_{k} \text { for any } k=0,1,2, \cdots, \text { i.e., } \\
d_{\alpha}\left(T_{k} x, T_{k} y\right) \leq \phi_{\alpha}\left(d_{j(\alpha)}(x, y)\right) .
\end{gathered}
$$

If $\left\{T_{k}\right\}_{k=1}^{\infty}$ converges pointwise to $y_{0}$, then the sequence $\left\{y_{k}\right\}_{k=1}^{\infty}$ converges to $y_{0}$.

\section{Applications}

Here we shall apply the results obtained to some initial value problems considered in [2].

Let us consider the initial value problems

$$
\begin{aligned}
& \varphi^{\prime}(t)=F_{k}\left(t, \varphi\left(\Delta_{1}(t)\right), \ldots, \varphi\left(\Delta_{m}(t)\right), \varphi^{\prime}\left(\tau_{1}(t)\right), \ldots .\right. \\
& \left.\varphi^{\prime}\left(\tau_{n}(t)\right)\right)
\end{aligned}
$$

$$
t>0, \varphi(t)=\psi(t), \varphi^{\prime}(t)=\psi^{\prime}(t), t \leq 0,
$$

where $\varphi(t)$ is the unknown function. The deviations $\Delta_{i}(t)=\tau_{l}(t), \quad i=1,2, \ldots ., m ; l=1,2, \ldots, n$

are of mixed type and in general case unbounded. After usual transformations, assuming

$\psi(t)=0$ equation $\left(4_{k}\right)$ can be reduced to the following one $\left(x(t)=\varphi^{\prime}(t)\right)$ for $t>0$ and $\theta(t)=\psi^{\prime}(t)$ for $t \leq 0$ :

$$
\begin{aligned}
& x(t)=F_{k}\left(t, \int_{0}^{\Delta_{1}(t)} x(s) d s, \ldots, \int_{0}^{\Delta_{m}(t)} x(s) d s, x\left(\tau_{1}(t)\right), \ldots,\right. \\
& \left.x\left(\tau_{n}(t)\right)\right), \quad t>0, x(t)=\theta(t), t \leq 0
\end{aligned}
$$

Let $C\left(R^{1}\right)$ be the linear topological space consisting of all continuous function $f(t): R^{1} \rightarrow R^{1}$ with a topology generated by a saturated family of seminorms $A=\{\|\| K\},.\|f\| K=\sup \{|f(t)|: t \in K\}$ where $k \subset R^{1}$ runs over all compact subsets of $R^{1}$. In view of Theorem 2 we shall look for a solution of $\left(5_{k}\right)$ in a locally compact set of functions.

Namely, let us consider the set

$c_{L}=\left\{f \in C\left(R^{1}\right):|f(t)-f(\bar{t})| \leq L|t-\bar{t}|\right.$ for every

$\left.t, \bar{t} \in R^{1}\right\}$, where the Lipschitz constant $L$ does not depend on $K$. It is easy to verify that $C_{L}$ is closed convex and every point has a neighbourhood with a compact closure. We shall find a solution of $\left(5_{k}\right)$ in the set

$C_{L}{ }^{0}=\left\{f \in C_{L}:|f(t)| \leq r_{0}(t)\right\}$ where $r_{0}(t): R^{1} \rightarrow R_{+}^{1}$

, $r_{0}(t)$ is continuous positive function on $R^{1}$

We shall make the following assumption [2]

$\Delta_{i}(t), \tau(t): R_{+}^{1} \rightarrow R^{1}\left(R_{+}^{1}=[0, \infty)\right)$ are continuous

(C1) $\Delta_{i}(0) \leq 0, \tau_{1}(0) \leq 0$

and $\left|\nabla_{i}(t)-\nabla_{i}(\bar{t})\right| \leq P_{i}|t-\bar{t}|$,

$\left|\tau_{1}(t)-\tau_{1}(\bar{t})\right| \leq Q_{1}|t-\bar{t}|$.

The map $j: A \rightarrow A$ is defined as in [2], where the index set $A$ consists all of compact subsets of $R^{1}$ :

(C2) For every $k=0,1,2,3, \ldots$ the functions $F_{k}\left(t, u_{1}, \ldots . u_{m}, v_{1}, \ldots v_{n}\right): R_{+}^{1} \times R^{m n} \rightarrow R^{1}$ are continuous and satisfy conditions:

(C3) $\left|F_{k}\left(t, u_{1}, \ldots . . u_{m}, v_{1}, \ldots . v_{n}\right)\right| \leq w(t)\left[1+\sum_{i=1}^{m}\left|u_{i}\right|+\sum_{l=1}^{n}\left|v_{l}\right|\right]$ (C4) $\begin{aligned} & \left|F_{k}\left(t, u_{1}, \ldots . . u_{m}, v_{1}, \ldots . v_{n}\right)-F_{k}\left(t, \bar{u}_{1}, \ldots . . \bar{u}_{m}, \bar{v}_{1}, \ldots . \bar{v}_{n}\right)\right| \leq \\ & \Omega\left[\left|u_{1}-\bar{u}_{1}\right|+\ldots . .+\left|u_{m}-\bar{u}_{m}\right|+\left|v_{1}-\bar{v}_{1}\right|+\ldots . .+\left|v_{m}-\bar{v}_{m}\right|\right]\end{aligned}$

where $\Omega$ is a positive constant:

$\left|F_{k}\left(t, u_{1}, \ldots . u_{m}, v_{1}, \ldots . v_{n}\right)-F_{k}\left(\bar{t}, u_{1}, \ldots . u_{m}, v_{1}, \ldots . v_{n}\right)\right| \leq$ $L_{0}|t-\bar{t}|$

where $L_{0}$ is a positive constant and

$L_{0}+\Omega\left[r_{0}(t) \sum_{i=1}^{m}\left|P_{i}\right|+\sum_{l=1}^{n}\left|Q_{l}\right|\right] \leq L$ 


$$
\begin{gathered}
w(t)\left[1+\sum_{i=1}^{m}\left|\Delta_{i}(t)\right| r_{0}(t)+n r_{0}(t)\right] \leq . r_{0}(t) ; \\
\Omega\left(m \bar{\Delta}_{k}+n\right)<1
\end{gathered}
$$

for every compact $K \subset R^{1}$ where $\bar{\Delta}_{k}=$ sup

$\{|\Delta(t)|: t \in K\}$. Conditions (C3) and (C4) are the same as in [2], assuming that the initial functions have Lipschitz constants

Theorem 3. Let the assumptions (C1) - (C4) be fulfilled. If the sequence of functions $\left\{F_{K}\right\}_{K=1}^{\infty}$ tends pointwise to $F_{0}$, then the sequence of solutions of $\left(5_{K}\right)$ tends to the solution of $\left(5_{0}\right)$.

Proof. We form by the right hand side of $\left(5_{K}\right)$ the sequence of operators $\left\{T_{K}\right\}_{K=1}^{\infty}$.

It is easy to see that $T_{K}$ maps the set $C_{L}^{0}=\left\{f \in C_{L}\left(R^{1}\right):|f(t)| \leq r_{0}(t), t \geq 0\right\}$ into itself. We shall verify only that $\left\{T_{K} f\right\}(t)$ has a Lipschitz constant equals to $L$, because another details of the proof are as in [2]. For $t, \bar{t}>0$ we have

$$
\begin{aligned}
& \left|\left(T_{k} f\right)\left(t-\left(T_{k} f\right)\right)(\bar{t})\right| \leq L_{0}|t-\bar{t}|+ \\
& \Omega\left[r_{0}(t) \sum_{i=1}^{m}\left|\Delta_{i}(t)-\Delta_{i}(\bar{t})\right|+\sum_{l=1}^{n} L\left|\tau_{l}(t)-\tau_{l}(\bar{t})\right|\right] \leq \\
& L_{0}|t-\bar{t}|+\Omega\left[r_{0}(t) \sum_{i=1}^{m} P_{i}+L \sum_{l=1}^{n} Q_{l}\right]|t-\bar{t}| \leq L|t-\bar{t}|
\end{aligned}
$$

Now we can apply Theorem 2 in order to conclude that the solution of $\left(5_{K}\right)$ tends to the solution of $\left(5_{0}\right)$. This is possible because $T_{K}$ is an equicontinuous family of operators and then pointwise convergence on compact sets implies a uniform convergence.

\section{Conclusion}

The stability of the fixed point sets of a uniformly convergent sequence of set-valued contractions is proved under the assumption that the maps are defined on a closed bounded subset $B$ of Hilbert space and take values in the family of non-empty closed convex subsets of $B$.

The convergence of sequence of fixed points of a convergent sequence of set-valued contractions can be easily investigated in a metric space setting. By restricting the underlying space to be a Hilbert space we prove the convergence of the sequence of fixed point sets of a convergent sequence of set-valued contractions. This also extends a similar result for point valued maps [4] to the set valued case.

1. Angelov, V. G., 1992, A continuoum dependence of fixed points of $\phi$-contractive mappings in uniform spaces, Archivum Mathematicum (BRNO), 28, 155-162.

2. Angelov, V.G., 1987, Fixed point theorems in uniform spaces and applications, Czechoslobvac Math. J. 37, 19-33.

3. Bonsal, F.F, 1962, Lectures on some fixed point theorems of functional analysis, Tata Institute of Fundamental Research Bombay, India

4. Die'udonne',J. 1960, Foundation of modern analysis, Pure and Appl. Math. Vol.10, Academic press, New York,. M.R 22, 11074.

5. Filippov, A.,1967, Classical solutions of differential equations with multi-valued right hand side, SIAM, J. Control 5609621. MR 36, 4047.

6. Kelley, J., 1959, General Topology, D.Van Nostrand Company, New York.

7. Markin, J.T, 1978, A fixed point stability theorem for nonexpansive set-valued mappings, Jour. Math. Anal. Appl. 54, 441-443.

8. Markin, J.T, 1973, Continuous dependence of fixed point set, Proc. Amer. Math. Soc. 38, 545-547.

9. Nadler, S.B.Jr ,1969, Multi-valued contraction mappings, Pac. J. Math 475-488. 